\title{
Efeito da desvalorização cambial na oferta, no preço de insumos e na relação entre os fatores na cultura do café
}

\author{
Eduardo Rodrigues de Castro ${ }^{1}$ \\ Erly Cardoso Teixeira ${ }^{2}$ \\ João Eustáquio de Lima ${ }^{3}$
}

Resumo: Este trabalho objetivou avaliar o impacto da desvalorização do Real, ocorrida em 1999, no preço de insumos e na composição dos custos na cultura de café. Ao considerar a desvalorização cambial, o preço desses fatores aumentou, o que reduziu o efeito positivo da desvalorização cambial sobre a produção, em relação à análise que não considerou o impacto no preço dos insumos. A desvalorização cambial levou a aumentos de $19,75 \%$ na oferta quando não se considerou o impacto no preço dos insumos, e de $17,69 \%$, quando se considerou o impacto da desvalorização cambial no preço dos insumos e de máquinas.

Palavras chave: desvalorização cambial, função translog, café.

\section{Classificação JEL: D21}

Abstract: The objective of this paper is to analyze the 1999 exchange rate (real/dollar) devaluation impact on coffee and intermediate input prices. Input price increased with exchange rate devaluation, reducing the posi-

\footnotetext{
${ }^{1}$ Eng ${ }^{\circ}$-Agrônomo, doutorando em Economia Aplicada, Departamento de Economia Rural -Universidade Federal de Viçosa. edu2110@hotmail.com

${ }^{2} \mathrm{PhD}$, Professor Titular do Departamento de Economia Rural da Universidade Federal deViçosa. teixeira@ufv.br

${ }^{3}$ PhD, Professor Titular do Departamento de Economia Rural da Universidade Federal de Viçosa. jelima@ufv.br
} 
tive effect of the exchange rate on coffee production, compared with the scenario which did not take the impact on input price into account. Coffee supply increased by $19.75 \%$ when the exchange rate devaluation impact on input price was not considered and by $17.69 \%$ when the devaluation impact on the price of fertilizers and machinery was considered.

Keywords: Exchange rate devaluation, translog function, coffee business.

JEL Classification: D21

\section{Introdução}

Na década de 1990, o setor agrícola foi penalizado pela predominância de uma taxa de câmbio valorizada, na maior parte do período. No início da década, a abertura comercial reduziu tarifas e desvalorizou a moeda. A partir de 1994, com o plano Real, foi adotada a âncora cambial, que manteve o câmbio valorizado até 1999, quando houve desvalorização da moeda nacional diante do dólar.

A taxa de câmbio afeta a produção agrícola de duas formas, quais sejam, a desvalorização (valorização) cambial aumenta (reduz) a competitividade dos produtos no mercado internacional e, ao mesmo tempo, aumenta (reduz) o preço dos produtos importados, elevando (reduzindo) o custo de insumos que utilizam matéria-prima importada.

Com a desvalorização cambial, a redução do preço relativo dos bens exportáveis no mercado internacional levou ao aumento na competitividade desses produtos, embora com aumento nos custos de produção. No entanto, nos últimos anos, o estoque mundial de café tem apresentado tendência de aumento, com conseqüente redução nos preços recebidos pelos produtores (Coffeebusiness, 2001).

As alterações na política cambial têm grande influência nas culturas de exportação. Em 2002, foram produzidas 44.160 .150 sacas de café, das quais foram exportadas 22.813.281 sacas, gerando uma receita de exportação de US \$1.195,0 milhões, que representa 5,8\% das exportações do agronegócio e $2 \%$ das exportações totais (CNA, 2003).

As avaliações tradicionais para medir os efeitos da taxa de câmbio 
sobre a oferta do produto mostram que há um viés no sentido de aumentar a oferta estimada, por não levar em conta o efeito sobre os insumos importados. Na cultura do algodão, houve efeito médio de 2,53\% menor na oferta do produto, ao se considerar o efeito direto da desvalorização cambial sobre o preço dos insumos (Zylbersztajn 1983).

Figueiredo et al. (1999) ao trabalharem em um dos cenários empregados por Zylbersztajn (1983), avaliaram o resultado de choques cambiais na cultura do café na região de Viçosa (MG), e encontram efeitos positivos sobre a demanda de fatores e aumento da produção. Dentre os fatores, os maiores efeitos foram sobre terra, insumos e mão-de-obra, sendo a demanda de equipamentos pouco afetada, resultado que era esperado devido às condições topográficas da região.

O objetivo geral deste trabalho foi avaliar os impactos de choques na taxa de câmbio na cultura do café, utilizando o mesmo modelo aplicado por Figueiredo et al. (1999), mas aplicando-se choques resultantes da desvalorização cambial de 1999 sobre o preço de insumos e equipamentos. Utilizaram-se dados da cultura do café da região Sul de Minas Gerais, que apresenta características diferentes das condições do referido trabalho. Especificamente, serão estimadas as elasticidadespreço diretas e cruzadas da demanda, as elasticidades de substituição de Allen e as mudanças relativas na oferta, nos preços dos insumos, na quantidade demandada e nas parcelas de custo.

\section{Referencial Teórico}

Os preços das culturas exportáveis são formados no mercado internacional e são determinados pelo equilíbrio entre oferta e demanda. A oferta mundial é determinada pelos excedentes dos países produtores, enquanto a demanda mundial, pelo excesso de demanda nos países importadores, conforme pode ser observado na Figura 1. A partir da condição de equilíbrio no país exportador, inicia-se a curva de oferta do mercado internacional, enquanto a curva de demanda é determinada pelo equilíbrio do mercado interno do país importador. Com isso, o país exportador recebe um preço maior do que receberia caso vendesse apenas no mercado interno, enquanto o país importador paga um preço menor do que pagaria se dependesse apenas de sua produção interna. 
Figura 1 - Equilíbrio no mercado internacional de um produto exportável.

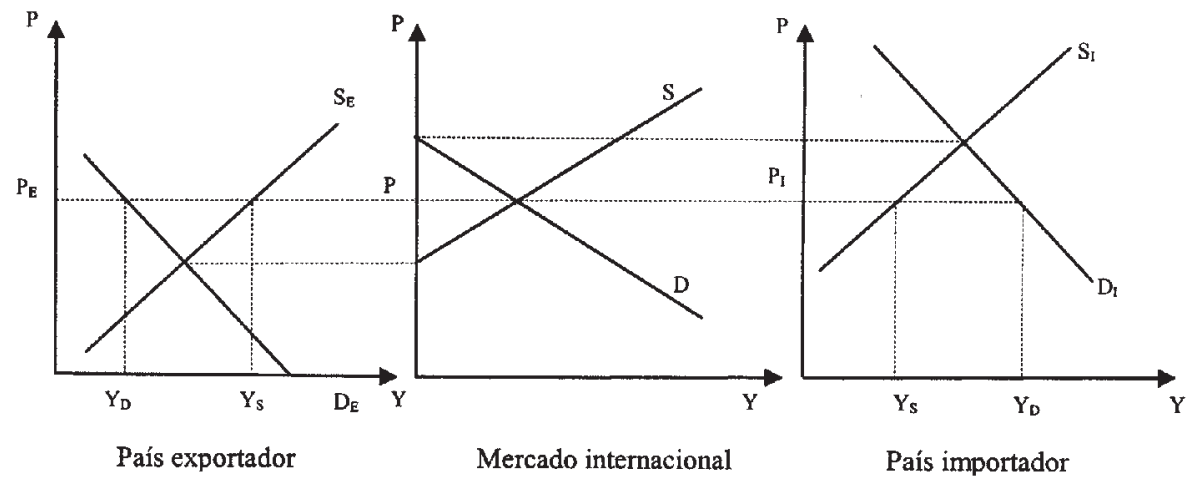

A desvalorização cambial causa efeitos diferentes sobre a cultura de exportação. Por um lado, aumento no preço dos fertilizantes resultaria na redução do consumo destes, ainda que o impacto não fosse repassado totalmente para os preços do insumo. A redução do consumo de fertilizantes levaria a um deslocamento da curva de oferta para a esquerda (1), com redução na produção de $Y_{S 1}$ para $Y_{S 2}$ (Figura 2). Por outro, a mesma desvalorização causaria aumento no preço interno do produto, de $P_{1}$ para $P_{2}$, levando a um aumento na oferta do produto para $Y_{S 3}$. Considerando-se que o país exportador não seja grande o suficiente para afetar o mercado internacional e que o aumento da oferta não teria reflexo nos preços internacionais, a exportação tornar-se-ia mais interessante para os produtores, de modo que estes iriam preferir vender no mercado externo que no interno. Com isso, o preço para o consumidor no país exportador, que, em princípio, não seria afetado pela desvalorização cambial, sofre um aumento e a demanda reduziria de $Y_{D 1}$ para $Y_{D 2}$.

Assim, o efeito final seria um deslocamento da curva de oferta do produto para a esquerda, provocado pelo aumento do preço dos insumos, e um deslocamento ao longo da curva de oferta, provocado pelo aumento no preço do produto no mercado interno (Figura 2). Se a desvalorização cambial causasse impacto apenas no preço do produto, a quantidade final seria $Y_{S 4}$ maior do que quando se considera o efeito da desvalorização sobre o preço dos insumos. 
Figura 2 - Efeito da desvalorização cambial na oferta do produto.

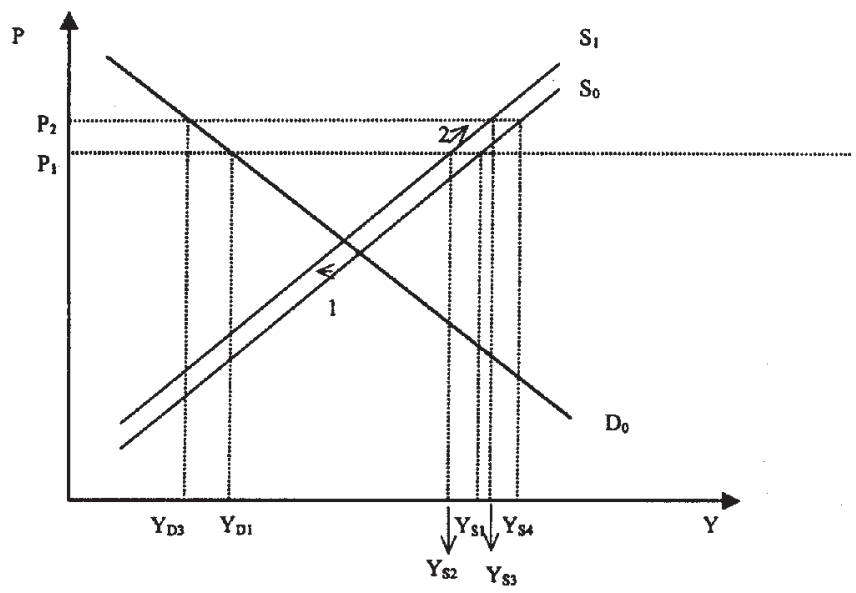

\section{Modelo Analítico}

Admitindo-se que uma firma atuasse em competição perfeita em ambos os mercados, de insumos e de produto, o processo produtivo poderia ser representado por uma função de produção linear homogênea: ${ }^{4}$

$$
Y=f\left(X_{1}, X_{2}, \ldots, X_{N}\right) \text {, }
$$

em que $Y$ é produto e $X_{i}(i=1,2, \ldots N)$, quantidade dos insumos.

Com base nos teoremas de dualidade, sob condições de regularidade, a função de produção tem uma representação dual dada pela função de custo:

$$
C=C(Y, W)
$$

em que $W$ é o vetor de preços exógenos dos insumos.

Ao aplicar o lema de Shephard, pode-se obter a demanda de fatores de produção condicionada pelo produto, tal como na equação (3):

$$
\frac{\delta C(Y, W)}{\delta W_{i}}=X_{I}(Y, W) .
$$

\footnotetext{
${ }^{4}$ Esta seção é baseada em Zylbersztajn (1983).
} 
Em condições de equilíbrio, a produção eficiente para a firma seria obtida no ponto onde o preço do produto se igualasse ao custo marginal, ou seja

$$
\frac{\delta Y C(W)}{\delta Y}=C(W)=P_{Y} .
$$

Diferenciando-se a equação (4), substituindo o resultado na equação (3) e reescrevendo-a em termos de mudanças relativas, obtém-se:

$$
E_{P y}=\sum S_{i} E_{w i}
$$

em que $\mathrm{S}_{i}$ é a parcela de custo do fator i; $E_{w i}$, mudança relativa no preço dos fatores; e $\mathrm{E}$, operador da mudança relativa $(E \xi=d \ln \xi)$.

O diferencial total da equação (3) e o conceito de elasticidade de substituição, de Allen Uzawa, levam à obtenção das equações de demanda derivada de fatores de produção, que pode ser expressa da seguinte forma:

$$
E_{X i}=\sum \sigma_{i j} S_{j}+E_{y}
$$

em que $E_{x i}$ é mudança relativa na quantidade de insumos; $\sigma_{i j}$, elasticidade parcial de substituição de Allen; e $\mathbf{E}_{\mathrm{y}}$ mudança relativa na quantidade de produto.

As equações que captam a mudança relativa no preço do produto e no preço dos insumos são, respectivamente,

$$
\begin{aligned}
& E_{P y}=\left(\frac{1}{\eta}\right) E_{y}+T_{y}, \\
& E_{w i}=\left(\frac{1}{\varepsilon_{i}}\right) E_{x i}+T_{x},
\end{aligned}
$$

em que $E_{P y}$ é mudança relativa no preço do produto; $E_{w i}$, mudança relativa no preço do insumo; $\eta$, elasticidade-preço da demanda do produto; $\varepsilon_{\mathrm{i}}$, elasticidade-preço direta da oferta do fator i ; $T_{y}$ e $T_{x}$, deslocadores verticais das funções de demanda de produto e oferta de fatores, expressas em mudanças percentuais. Os deslocadores verticais são os vetores nos quais serão aplicados os choques de demanda. 
O sistema de equações (9) a (11) relaciona os mercados de insumos com o produto e contém $E_{W}, E_{X}, E_{Y}$, variáveis endógenas.

Construindo as matrizes de elasticidades preço da oferta, $(\Gamma)$ e demanda dos fatores $(\Phi)$, e ainda um vetor de parcelas de custo $(S)$, obtêm-se as seguintes equações:

$$
E_{y}=\left[S^{\prime}(\Gamma-\Phi)^{-1} \gamma\right]^{-1} T y-\left[S^{\prime}(\Gamma-\Phi)^{-1} \gamma\right]^{-1}\left[S^{\prime}(\Gamma-\Phi)^{-1}\right] \delta T x(9)
$$

em que $(\Gamma-\Phi)^{-1}=\left(1-\Gamma^{-1} \Phi\right)^{-1} \Gamma^{-1} ; \delta$ é um vetor de zeros e uns, que permite introduzir choques nos preços de qualquer fator; e $\gamma$, vetor coluna de uns.

A mudança relativa no preço dos fatores é dada por:

$$
\begin{aligned}
& E_{w}=(\Gamma-\Phi)^{-1} \gamma D^{-1} T_{y}+\left\{\left[I-(\Gamma-\Phi)^{-1} \gamma D^{-1} S^{\prime}\right](\Gamma-\Phi)^{-1} \Gamma\right\} \delta T_{x,(10)} \\
& \text { em que } \mathrm{D}=\mathrm{S}^{\prime}(\Gamma-\Phi)^{-1} \gamma \\
& \quad \text { A mudança relativa na quantidade dos fatores é dada por: } \\
& E_{x}=\left[\Phi(\Gamma-\Phi)^{-1} \gamma D^{-1}+\gamma D^{-1}\right] T_{y}+ \\
& \left\{\Phi\left[I-(\Gamma-\Phi)^{-1} \gamma D^{-1} S^{\prime}\right]\left[(\Gamma-\Phi)^{-1} \Gamma\right]-\gamma D^{-1}\left[S^{\prime}(\Gamma-\Phi)^{-1} \Gamma\right]\right\} \delta T,(11)
\end{aligned}
$$

e, por fim, a mudança relativa nas parcelas:

$$
E_{S_{j}}=E_{w_{j}}+E_{x_{j}}-E_{P y}-E_{y^{\prime}} \text {. }
$$

\section{Estimativa dos parâmetros ${ }^{5}$}

A função de custo transcendental logarítmica (translog) é uma função flexível, visto que não impõe restrições a priori à função de produção a ela associada.

Tomando-se a função de custo médio, obtida a partir de (2), aplicando-se logaritmos naturais à expressão e fazendo-se a expansão, por meio de uma série de Taylor, em torno do vetor unitário, obtém-se a função translog de custo, representada pela expressão (13):

$$
\ln C^{*}=\beta_{0}+\sum_{i}^{n} \beta_{i} \ln P_{i}+\frac{1}{2} \sum_{i}^{n} \sum_{j}^{n} \beta_{i j} \ln P_{i} \ln P_{j},
$$

em que os parâmetros estruturais são representados por $\beta_{\mathrm{s}}$; $P$ é o preço;

\footnotetext{
${ }^{5}$ Baseado em Hertel, 1984.
} 
e os índices $i$ e $j$ identificam os fatores de produção utilizados na estimação da função custo - Mão-de-Obra (M); Equipamentos (E); Insumos (I) e Terra (T), cujas unidades de medida são dias-homem, horas de trator, quilos e hectares, respectivamente. Para que satisfaça as propriedades teóricas da função de custo, a equação (2) deve ser contínua e diferenciável nos preços, obedecer às condições de simetria e homogeneidade (homogênea de grau um nos preços dos fatores) e ainda estabelecer as restrições de monotonicidade positiva e de quase concavidade nos preços dos fatores. A condição de simetria é imposta pela restrição:

$\beta_{i j}=\beta_{\mathrm{ji}}$ para $\mathrm{i} \neq \mathrm{j}$,

e a homogeneidade linear é garantida pelas condições:

$$
\sum_{i=1}^{n} \beta_{i}=1 \text { e } \sum_{i=1}^{n} \beta_{j}=\sum_{j=1}^{n} \beta_{j}=0 .
$$

Para a função Translog, as condições de monotonicidade e concavidade são conferidas localmente. A monotonicidade da função é condicionada pelo comportamento das parcelas de custo e será atendida se estas apresentarem sinal não-negativo, isto é, maior que zero, já que o aumento do uso dos fatores provoca elevação dos custos. Para que a concavidade seja satisfeita, é necessária que a matriz hessiana orlada seja negativa semidefinida.

Tomando-se a derivada parcial da expressão (2), em relação a todos os preços dos fatores, e fazendo uso do Lema de Shephard, obtém-se um sistema de $n$ equações, que representam as demandas derivadas dos fatores de produção em função dos preços. No entanto, ao derivar parcialmente a expressão (13) em relação a $\ln P_{i}$, obtém-se a equação das parcelas de custo:

$$
\frac{\delta \ln C}{\delta \ln P_{i}}=\frac{\delta C}{\delta P_{i}} \frac{P_{i}}{C}=\frac{X_{i} P_{i}}{C}=S_{i}=\beta_{i}+\sum \beta i_{j} \ln P_{j},
$$

em que $\frac{\delta C}{\delta P_{i}}=X_{i}$, demanda derivada do fator $X ; S_{i}$, parcela de custo referente ao fator $i$. Assim, os parâmetros da função custo Translog podem ser determinados pela estimação de $n$ parcelas de custo que compõem o sistema de equações, representado por (14). 
As equações (15) representam as equações de parcelas de custo para cada fator a serem estimadas, normalizadas pelo preço do fator Terra:

$$
\begin{gathered}
S_{M}=\beta_{M}+\beta_{M M} \ln \frac{P_{M}}{P_{T}}+\beta_{M E} \ln \frac{P_{E}}{P_{T}}+\beta_{M I} \ln \frac{P_{I}}{P_{T}}+\varepsilon, \\
S_{E}=\beta_{E}+\beta_{M E} \ln \frac{P_{M}}{P_{T}}+\beta_{E E} \ln \frac{P_{E}}{P_{T}}+\beta_{E I} \ln \frac{P_{I}}{P_{T}}+\varepsilon, \\
S_{I}=\beta_{I}+\beta_{M I} \ln \frac{P_{M}}{P_{T}}+\beta_{I E} \ln \frac{P_{E}}{P_{T}}+\beta_{I I} \ln \frac{P_{I}}{P_{T}}+\varepsilon,
\end{gathered}
$$

Ao fazer a normalização, os parâmetros da equação da parcela do fator Terra podem ser estimados residualmente; nesse caso, não se teria o nível de significância estatística deles. Para contornar esse problema, é elaborado um novo sistema de equações, normalizado agora pelo preço de outro insumo. As características de homogeneidade linear da função indireta de custo é imposta, ao se fazer a soma das parcelas igual a $1\left(\sum_{i} S_{i}=1\right)$.

As elasticidades-preço diretas e cruzadas, representadas respectivamente por $\eta_{i i}$ e $\eta_{\mathrm{ij}}$, podem ser obtidas pelas expressões (16):

$$
\eta_{i i}=\frac{\beta_{\mathrm{ii}}}{\mathrm{S}_{\mathrm{i}}}+\mathrm{S}_{\mathrm{i}}-1, \quad \eta_{i j}=\frac{\beta_{i j}}{\mathrm{~S}_{\mathrm{i}}}+\mathrm{S}_{\mathrm{j}} .
$$

A partir das elasticidades preço direta e cruzada é possível obter as elasticidades de substituição de Allen, que são simétricas e são obtidas pelas expressões (17):

$$
\sigma_{i i}=\frac{\beta_{\mathrm{ii}}}{\mathrm{S}_{\mathrm{i}}^{2}}-\frac{1}{\mathrm{~S}_{\mathrm{i}}}+1, \quad \sigma_{i j}=\frac{\beta_{\mathrm{i} j}}{\mathrm{~S}_{\mathrm{i}} S_{i}}+1 .
$$

Os níveis de significância das elasticidades foram avaliados por meio do erro-padrão, obtido a partir dos erros-padrão dos coeficientes estimados:

$$
e p_{\eta_{i j}}=\frac{e p_{\beta_{i j}}}{S_{i}} ; \quad e p_{\eta_{i i}}=\frac{e p_{\beta i i}}{S_{i}} ;
$$




$$
e p_{\sigma i j}=\frac{e p_{\beta_{i j}}}{S_{i} S_{j}} ; \quad \quad e p_{\sigma i i}=\frac{e p_{\beta i i}}{S_{i}^{2}}
$$

\section{Teste de Separabilidade}

O conceito de separabilidade foi desenvolvido, inicialmente, para estudar problemas relativos à consistência na agregação de insumos e preços na teoria da demanda e produção. A definição de separabilidade, segundo Sono-Leontief, citado por Hertel (1984) requer que a taxa marginal de substituição entre duas variáveis $i$ e $j$ seja independente do nível da variável $k$ empregada, assegurando que os insumos $i$ e $j$ sejam separáveis de $k$, podendo ser agregados. Pelo teorema de Hotelling, isto significa que a razão ótima dos insumos $i$ e $j$ não depende do nível de preços da variável $k$. Berndt e Christensen (1973) desenvolveram uma relação entre o conceito de separabilidade e a elasticidade de substituição Allen-Uzawa. Nesse caso, o teste para verificar se os insumos $i$ e $j$ são separáveis de um terceiro insumo $k$ consiste em verificar se a elasticidade de substituição entre $i$ e $k$ é igual à elasticidade de substituição entre $j$ e $k\left(\sigma_{i k}=\sigma_{j k}\right)$. Como o objetivo deste trabalho é avaliar o efeito direto da desvalorização cambial sobre o preço dos insumos e, indiretamente, sobre as relações entre os outros fatores de produção, o teste visou avaliar a separabilidade dos fatores Mão-de-Obra, Equipamentos e Terra de Insumos. No entanto, parte-se do princípio que os fatores agregados em cada uma dessas variáveis sejam separáveis e, portanto, agregáveis dentro de cada uma das variáveis consideradas.

\section{Fonte de Dados}

Os choques considerados no modelo foram baseados no período de 1999 a 2001, no qual ocorreram mudanças no regime cambial, com forte desvalorização do Real. Assim, foram tomados os valores relativos médios da taxa de câmbio efetiva em 1999, 2000 e 2001, e calculada a desvalorização média ocorrida entre 1999-2000 e 2000-2001, chegando-se ao valor de $\mathrm{T}_{\mathrm{y}}$ (deslocador da oferta) de 10,75\%. O mesmo foi feito para encontrar o valor de $\mathrm{T}_{\mathrm{x}}$ (deslocador da demanda), tomando- 
se como base o Índice Geral no Atacado (IPA-OG da Fundação Getúlio Vargas) para fertilizantes, e calculando-se a variação média do período da mesma forma, chegando-se ao valor de 14,11\% .

Foram considerados três cenários: no primeiro, foi dado o choque na taxa de câmbio sem considerar seu impacto na demanda de insumos; no segundo, foi acrescentado o choque na demanda de fertilizantes, a exemplo do que foi feito por Figueiredo et al. (1999); no terceiro, foi considerado também o choque na demanda de equipamentos, uma vez que a desvalorização na taxa de câmbio provoca elevação do preço de combustíveis. O terceiro cenário foi acrescentado pelo fato de a cafeicultura no Sul de Minas apresentar maior nível de mecanização do que na Zona da Mata.

Foram utilizados, neste estudo, dados de série temporal de 1990 a 2001, obtidos das planilhas de custo de produção levantados pela COOPARAÍSO, localizada em São Sebastião do Paraíso, Minas Gerais. Na safra 2002/2003, a produção de Minas Gerais representou cerca de 51,8\% da produção nacional de café beneficiado, sendo 52,5\% produzido na região sul de Minas (ABIC, 2005). O município de São Sebastião do Paraíso foi o nono município em produção, no ano de 2002, e sua cooperativa atende a outros municípios da região (CoffeeBusiness, 2004).

O sistema de produção de café adotado foi o de solo com média fertilidade, considerando-se uma produtividade de 20 sacos de $60 \mathrm{~kg}$ de café beneficiado por hectare, uma vez que a produtividade média do Brasil, em 2002, foi de 18,63 sc/ha (Coffee Business, 2004)

Todos os valores e preços, no período de 1990 a 2001, foram atualizados para preços de outubro de 2002, pelo Índice Geral de Preços (IGP-DI), da Fundação Getúlio Vargas (FGV).

As parcelas de custo dos fatores de produção são representadas pelas despesas totais com cada um deles. Na parcela Trabalho foram considerados os serviços manuais para tratos culturais (adubação do solo, aplicação de defensivos, capinas manuais, desbrotas, arruação, aplicação de calcário) e mão-de-obra para colheita. O preço relativo à mão-de-obra foi o valor do dia de serviço pago na região. Nas despesas com máquinas foram consideradas as despesas com trator e com beneficiamento e secagem. Como proxy do preço desses serviços foi utilizada a hora de trator. Para a variável Insumo foram consideradas 
despesas com fertilizantes, defensivos agrícolas e demais insumos. No caso da variável Terra, foi utilizado o valor do aluguel anual para o Estado de São Paulo. Em todas as variáveis foram consideradas as despesas por hectare.

As elasticidades-preço da oferta para os insumos Mão-de-Obra, Equipamentos, Insumos e Terra utilizados foram calculadas por Lopes (1977), citado por Zylberstajn (1983)

Na estimação dos parâmetros foi utilizado o software Eviews, ${ }^{\circ}$ versão 3.1, pelo método de equações aparentemente não-relacionadas ( SUR ).

\section{Resultados e discussão}

Os valores dos coeficientes estimados para a função Translog encontram-se na Tabela 1. As condições de simetria foram impostas ao se estimar o modelo e podem ser verificadas pelos valores dos coeficientes. Os sinais encontrados são condizentes com a teoria, e apenas cinco deles não foram estatisticamente significativos a $10 \%$ de probabilidade. A partir desses coeficientes foram calculadas as elasticidades-preço da demanda e as elasticidades parciais de substituição de Allen, cujos valores se encontram nas Tabelas 2 e 3, respectivamente.

A Tabela 2 apresenta as elasticidades-preço da demanda, a partir da qual se obtiveram as elasticidades parciais de substituição de Allen (Tabela 3). As elasticidades-preço diretas relativas à mão-de-obra, insumos e terra, apesar de apresentarem sinal condizente com a teoria, foram as únicas não-significativas estatisticamente. As condições de homogeneidade impostas ao modelo, podem ser verificadas pela soma das linhas igual a zero. A concavidade nos preços da função custo pode ser observada pelo sinal negativo das elasticidades preço-direta, que se encontram na diagonal principal. As condições de monotonicidade também foram atendidas, uma vez que as parcelas estimadas apresentaram valor maior que zero. Os valores médios das parcelas são 0,4426, 0,0808, 0,4217, 0,055 para mão-de-obra, equipamentos, insumos e terra, respectivamente. 
Tabela 1- Estimativa dos parâmetros das parcelas de custo dos fatores de produção de café, no período de 1990-2001, Minas Gerais

\begin{tabular}{ccc}
\hline Parâmetros & Estimativa & Erro-Padrão \\
\hline$\beta_{M}$ & $-0,224654^{*}$ & 0,094929 \\
$\beta_{M M}$ & $0,221903^{*}$ & 0,032708 \\
$\beta_{M E}$ & $0,044886^{*}$ & 0,01376 \\
$\beta_{M I}$ & $-0,231502^{*}$ & 0,025954 \\
$\beta_{M T}$ & $-0,040895^{*}$ & 0,009238 \\
$\beta_{E}$ & $0,118136^{* * *}$ & 0,061624 \\
$\beta_{E M}$ & $0,044886^{*}$ & 0,01376 \\
$\beta_{E E}$ & $-0,019447^{* * *}$ & 0,010864 \\
$\beta_{E I}$ & $-0,010038^{\text {n.s. }}$ & 0,013432 \\
$\beta_{E T}$ & $-0,018009^{*}$ & 0,005588 \\
$\beta_{I}$ & $1,187649^{*}$ & 0,124685 \\
$\beta_{I M}$ & $-0,231502^{*}$ & 0,025954 \\
$\beta_{I E}$ & $-0,010038^{\text {n.s. }}$ & 1,000 \\
$\beta_{I I}$ & $0,241205^{*}$ & 0,022161 \\
$\beta_{I T}$ & $0,005244^{\text {n.s. }}$ & 0,009 \\
$\beta_{T}$ & $-0,062164^{\text {n.s. }}$ & 0,040124 \\
$\beta_{T M}$ & $-0,040895^{*}$ & 0,009238 \\
$\beta_{T E}$ & $-0,018010^{*}$ & 0,005588 \\
$\beta_{T I}$ & $0,005244^{\text {n.s. }}$ & 0,009 \\
$\beta_{T T}$ & $0,049997^{*}$ & 0,005502 \\
\hline & & \\
\hline
\end{tabular}

Fonte: Resultados da pesquisa.

n.s. : não-significativo estatisticamente.

* : estatisticamente significativo a $1 \%$.

** : estatisticamente significativo a $5 \%$.

*** : estatisticamente significativo a $10 \%$.

$\beta_{I T}=\beta_{T I}$ (condição de simetria).

$\beta_{i}$ é o intercepto da equação de parcela de custo $i$.

$\beta_{i j}$ é o parâmetro da variável ln $\left(\mathrm{P}_{\mathrm{j}} / \mathrm{P}_{\mathrm{k}}\right)$ na equação parcela de custo do insumo $i$.

$i, j$ denotam os fatores: $\mathrm{M}=$ Mão-de-Obra; $\mathrm{E}=$ Equipamentos; $\mathrm{I}=$ Insumos; $\mathrm{T}=$ Terra. 
Tabela 2 - Estimativa das elasticidades-preço direta e cruzada da demanda de fatores de produção de café, no período de 1990-2001, Minas Gerais (erros-padrão entre parênteses)

\begin{tabular}{c|c|c|c|c|c}
\hline \multicolumn{6}{c}{ Preço } \\
\hline Quantidade & Mão-de-Obra & Equipamento & Insumos & Terra & linhas \\
\hline Mão-de-Obra & $-0,0560^{\text {n.s. }}$ & $0,1823^{*}$ & $-0,1010^{* * *}$ & $-0,0380^{* * *}$ & $-0,0127$ \\
& $(0,0740)$ & $(0,0311)$ & $(0,0587)$ & $(0,0209)$ & \\
Equipamento & $0,9976^{*}$ & $-1,1598^{*}$ & $0,2982^{* * *}$ & $-0,1683^{* *}$ & $-0,0323$ \\
& $(0,1702)$ & $(0,1344)$ & $(0,1662)$ & $(0,0691)$ & \\
Insumos & $0,1057^{* * *}$ & $0,0571^{* *}$ & $-0,0066^{\text {n.s. }}$ & $0,0669^{*}$ & 0,0116 \\
& $(0,0614)$ & $(0,0238)$ & $(0,0525)$ & $(0,0213)$ & \\
Terra & $-0,3086^{* * *}$ & $-0,2498^{*}$ & $0,5187^{*}$ & $-0,0276^{\text {n.s. }}$ & $-0,0673$ \\
& $(0,1696)$ & $(0,1026)$ & $(0,1652)$ & $(0,1010)$ & \\
\hline
\end{tabular}

Fonte: Resultados da pesquisa.

n.s. : não-significativo estatisticamente.

* : estatisticamente significativo a $1 \%$.

** : estatisticamente significativo a $5 \%$.

*** : estatisticamente significativo a $10 \%$.

De acordo com a Tabela 3, os fatores mão-de-obra-equipamento, insumos-equipamento e insumos-terra são substitutos. A relação de substitutibilidade entre trabalho e equipamentos era esperada; entre insumos e equipamentos é justificada pelo fato de alguns tratos culturais na cultura do café podem ser realizados por máquinas ou herbicidas, que fazem parte da variável insumo. Zylberstajn (1983) e Figueiredo et al. (1999) acharam a mesma relação entre estas variáveis.

A relação de substituição terra-insumos não era esperada e indica que redução de $1 \%$ no preço da terra causou uma diminuição de 1,23\% na utilização de insumos. Este resultado pode ser conseqüência da metodologia utilizada, que trabalha com série de preços dos insumos, custo total, sendo a elasticidade de substituição função das elasticidadespreço direta da demanda e das parcelas de custo. No período analisado, a parcela relativa a insumos no custo total de produção caiu, e o mesmo ocorreu com o preço do fator terra, situação que levou ao resultado encontrado. Na realidade, o que aconteceu foi redução na área plantada, na década de 1990, ao mesmo tempo em que os preços da terra utilizadas no trabalho também apresentaram tendência de queda. O preço utilizado no fator terra foi o preço médio do aluguel de terra no Estado 
de São Paulo (devido à disponibilidade de dados), razão que este preço pode não refletir o valor real da terra para a cultura do café.

Os fatores terra-mão-de-obra, terra-equipamento e insumos-mãode-obra foram complementares, o que era esperado. Isto significa que, ao aumentar a demanda do fator terra, por exemplo, aumenta a demanda de mão-de-obra e equipamentos, e o mesmo ocorre para insumos e mão-de-obra.

Tabela 3 - Estimativa das elasticidades de substituição de Allen entre fatores de produção de café, no período de 1990-2001, Minas Gerais (erros-padrão entre parênteses)

\begin{tabular}{l|c|c|c|c}
\hline & Mão-de-obra & Equipamentos & Insumos & Terra \\
\hline Mão-de-obra & $-0,1266^{\text {n.s. }}$ & $2,2556^{*}$ & $-0,2391^{* * *}$ & $-0,6977^{* * *}$ \\
& $(0,1672)$ & $(0,3849)$ & $(0,1389)$ & $(0,3835)$ \\
Equipamentos & & $-14,3489^{*}$ & $0,7060^{* * *}$ & $-3,0909^{* *}$ \\
& & $(1,6629)$ & $(0,3934)$ & $(1,2494)$ \\
Insumos & & & $-0,0156^{\text {n.s. }}$ & $1,2279^{*}$ \\
& & & $(0,1242)$ & $(0,3912)$ \\
Terra & & & & $-0,5060^{\text {n.s. }}$ \\
& & & & $(1,8548)$ \\
\hline
\end{tabular}

Fonte: Resultados da pesquisa

n.s. : não significativo estatisticamente.

* : estatisticamente significativo a $1 \%$.

** : estatisticamente significativo a $5 \%$.

*** : estatisticamente significativo a $10 \%$.

A Tabela 4 apresenta os resultados, em valores percentuais, do efeito da taxa de câmbio nas variáveis consideradas, na forma de mudança relativa na quantidade dos insumos $\left(E_{x}\right)$, no preço dos insumos $\left(E_{w}\right)$, nas parcelas de custo $\left(S_{i}\right)$ e na quantidade ofertada $\left(E_{y}\right)$.

Na coluna (1) tem-se o cenário 1 , em que $\mathrm{T}_{\mathrm{x}}=0$, ou seja, os efeitos da desvalorização cambial incidem apenas sobre o preço do produto. Na coluna (2) tem-se o cenário 2, em que se considerou o impacto de $14,11 \%$ no preço dos insumos. A terceira coluna é a diferença entre os dois cenários (1-2). Na quarta coluna, tem-se o cenário 3, em que se considerou o mesmo impacto $(14,11 \%)$, mas que incidiu agora sobre as variáveis insumos e equipamentos. A quinta coluna é a diferença entre o cenário 1 e o cenário 3 (1-3). 
Tabela 4 - Mudanças relativas decorrentes dos choques cambiais exógenos, em \%, no período de 1990-2001, Minas Gerais

\begin{tabular}{c|c|c|c|c|c}
\hline & Cenário 1 & Cenário 2 & $(1-2)$ & Cenário 3 & $(1-3)$ \\
\hline Especificação & $T_{X=} 0$ & $T_{X}=14,11^{1}$ & Diferença & $T_{X}=14,11^{2}$ & Diferença \\
\hline$E_{X M}$ & 21,24 & 6,32 & 14,92 & 6,30 & 14,94 \\
$E_{X E}$ & 9,70 & 3,44 & 6,26 & $-1,49$ & 11,19 \\
$E_{X I}$ & 20,92 & 10,31 & 10,61 & 9,11 & 11,81 \\
$E_{X T}$ & 8,79 & 11,17 & $-2,38$ & 9,25 & $-0,46$ \\
\hline$E_{W M}$ & 17,27 & 5,14 & 12,13 & 5,12 & 12,15 \\
$E_{W E}$ & 19,40 & 6,88 & 12,53 & 11,12 & 8,28 \\
$E_{W I}$ & 0,00 & 14,11 & $-14,11$ & 14,11 & $-14,11$ \\
$E_{W T}$ & 28,36 & 36,03 & $-7,67$ & 29,85 & $-1,49$ \\
\hline$E_{S M}$ & 8,00 & $-19,04$ & 27,00 & $-17,02$ & 25,02 \\
$E_{S E}$ & $-1,00$ & $-20,18$ & 19,00 & $-18,81$ & 17,81 \\
$E_{S I}$ & $-10,00$ & $-6,08$ & $-3,00$ & $-5,22$ & $-4,78$ \\
$E_{S T}$ & 7,00 & 16,70 & $-10,00$ & 10,66 & $-3,66$ \\
\hline$E_{Y}$ & 19,75 & 19,75 & 0,00 & 17,69 & 2,06 \\
\hline
\end{tabular}

$E_{X i}$ é a mudança relativa na quantidade do fator $i$ ( $i=$ mão-de-obra, equipamentos, insumos e terra); $E_{W i}$ é a mudança relativa no preço dos fatores $i ; E_{S i}$ é a mudança relativa na parcela de custo dos fatores; e, $E_{Y}$ é a mudança relativa na oferta do café.

1 - Considerando-se o impacto da alteração da taxa de câmbio apenas sobre o preço dos insumos.

2- Considerando-se o impacto da alteração da taxa de câmbio sobre o preço dos insumos e sobre o preço de equipamentos.

Fonte: Resultados da pesquisa.

Observa-se que houve alteração nas variáveis consideradas, ao comparar o modelo tradicional com o modelo em que ocorre impacto da mudança cambial no preço dos insumos (cenário 1), que pode ser observado na coluna 3. Só não houve variação na oferta relativa do produto, ou seja, nos dois cenários houve aumento de $19,75 \%$ na oferta do produto, devido à desvalorização da taxa de câmbio. Isto ocorreu por causa do modelo empregado para medir esse efeito, dado pela equação (9). Ao considerar o efeito da desvalorização apenas sobre o preço dos insumos, verifica-se que o vetor $\delta$ (que é um vetor de zeros e uns) anulou os efeitos das outras variáveis. Como a elasticidade de oferta de fertilizantes foi considerada infinita, o efeito da variável insumos sobre a oferta do produto, mesmo no cenário 2 , não causou alteração na oferta, em relação ao cenário 1 . Ao considerar o efeito da desvalorização da taxa de câmbio sobre o preço de equipamentos (coluna 4), o aumento 
da oferta passou a ser 2,06\% menor do que o do cenário 1 (coluna 5). Zylbersztajn (1983), ao trabalhar com as culturas de algodão e milho, encontrou variações até $4,64 \%$ na oferta do produto e ressaltou que esses efeitos foram distintos em cada cultura, devido à diferença na utilização dos fatores de produção.

Ao comparar a coluna $5 \mathrm{com}$ a 3 , percebe-se que, ao considerar o impacto nos insumos e nos equipamentos, as mudanças relativas são ainda menores do que quando se considera o cenário 1. Esse resultado é importante quando se leva em conta que os dados utilizados representam um sistema de produção no qual o uso de equipamentos tem participação importante no custo de produção, já que a mecanização é mais intensiva e há maior participação no custo de produção, ao contrário da região analisada por Figueiredo et al. (1999). O choque na variável equipamentos é justificado, já que uma desvalorização cambial poderá provocar alteração no preço de combustível e refletir no preço dos serviços de máquinas.

Ao avaliar a mudança relativa das quantidades $\left(E_{x i}\right)$, pode-se observar que, ao considerar o cenário 2, a demanda de insumos foi menor do que a do cenário 1. Caso houvesse um aumento no preço de insumos, decorrente da desvalorização cambial, haveria queda na demanda desse fator, na de mão-de-obra e na de equipamentos, respectivamente de $10,61 \%, 14,92 \%$ e $6,26 \%$, uma vez que estes fatores são complementares. No caso do fator terra, no cenário 2, ao aumentar o preço dos insumos, a demanda de terra seria $2,38 \%$ maior do que a do cenário 1 , o que reforça a relação de substituição entre estas variáveis. No cenário 3 , ao efetuar o choque na variável equipamentos, reduziu-se a demanda desse fator. Quanto às outras variáveis, as mudanças relativas na demanda foram menores do que as do cenário 2 .

No caso da variação nos preços, o efeito só aparece quando se considera o cenário 2. Figueiredo et al. (1999), ao desconsiderarem o efeito da taxa de câmbio sobre o preço dos fertilizantes em seu trabalho $\left(\mathrm{T}_{\mathrm{x}}=\right.$ 0 ), encontraram resultado nulo. Nesse caso, é interessante notar que, se o impacto no preço de fertilizantes fosse nulo, o aumento da demanda desse elevaria a demanda dos fatores mão-de-obra e equipamentos. Com isso as mudanças relativas no preço desses fatores seria de $12,13 \%$ e $12,53 \%$ menores em relação ao cenário 1 . No cenário 2 quando os 
preços dos insumos se elevassem, a demanda desse fator reduziria, reduzindo também a demanda de mão-de-obra e equipamentos, o que refletiria em menor aumento de preços. Ao considerar o cenário 3, as mudanças relativas nos preços seriam ainda menores.

Com relação à mudança relativa nas parcelas, o aumento na oferta do produto provocou alteração na composição dos custos de produção de café. No cenário 1, foi maior a participação de terra e mão-de-obra no custo total. Isto pode ser explicado pela alta mudança relativa tanto na quantidade demandada $\left(E_{X M}\right.$ e $\left.E_{X T}\right)$ quanto na mudança relativa dos preços. Quando se consideram os cenários 2 e 3, verifica-se a mudança na quantidade demandada dos fatores mão-de-obra, equipamentos e insumos foi reduzida. A mudança relativa no preço desses fatores (com exceção de insumos) foi relativamente baixa, o que fez com que se reduzisse a parcela desses fatores no custo total. Nestes cenários, o fator terra foi o único que apresentou aumento na parcela de custos, em conseqüência do aumento na demanda e nos preços desse insumo. Este resultado também foi encontrado por Figueiredo et al. (1999), em que os preços e a demanda deste fator fizeram com que fosse o único fator a aumentar a participação após os choques. Deve-se lembrar que a mudança na parcela de custos pode ser conseqüência direta do aumento de preços e/ou aumento da quantidade utilizada do fator, quanto da mudança da participação de outros fatores. Por exemplo, o preço da variável insumos foi aumentado devido ao choque, no entanto sua parcela nos custos de produção caiu, em virtude do maior aumento na participação do fator terra.

Na Tabela 5 encontram-se os resultados do teste de separabilidade. Em primeiro lugar, foi feito o teste de separabilidade global completa, pelo qual, segundo Berndt e Christensen, (1973), verifica-se que $\beta_{M T}=$ $\beta_{E I}=\beta_{T I}=\beta_{M E}=\beta_{T E}=\beta_{M I}=1$, o que não ocorreu. Como não houve separabilidade completa, foi feito o teste de separabilidade linear, o qual consiste em verificar se as elasticidades são iguais a 1, o que ocorreu apenas para terra e equipamentos, que são separáveis de insumos. Quando as elasticidades forem diferentes de 1, faz-se o teste de separabilidade não-linear, que consiste em testar se as elasticidades são simplesmente iguais.

Esses testes são feitos no Wald Test, do programa E-views, de 
modo que, se P-valor menor que o nível de significância, rejeita-se a hipótese nula, ou seja, as variáveis consideradas não são separáveis.

Tabela 5 - Resultados do teste de separabilidade para as variáveis utilizadas, no período de 1990-2001, Minas Gerais

\begin{tabular}{c|c|c|c|c}
\hline Separabilidade & Teste linear & P valor & Teste não-linear & P valor \\
\hline M,E,T de I & $\sigma_{M I}=\sigma_{E I}=\sigma_{T I}$ & & $\sigma_{M I}=\sigma_{E I}=\sigma_{T I}$ & 0,0000 \\
M,E de I & & & $\sigma_{M I}=\sigma_{E I}$ & 0,0255 \\
E,T de I & $\sigma_{M I}=\sigma_{E I}=1$ & 0,2929 & $\sigma_{M I}=\sigma_{E I}$ & 0,1176 \\
M,T de I & & & $\sigma_{M I}=\sigma_{T I}$ & 0,000 \\
E, T de M & & & $\sigma_{E M}=\sigma_{T M}$ & 0,000 \\
E,T,I de M & & & $\sigma_{E M}=\sigma_{I M}=\sigma_{T M}$ & 0,000 \\
\hline
\end{tabular}

Fonte: Resultados da pesquisa.

Este teste é baseado na estatística de quiquadrado $(\boldsymbol{\chi})$

Os testes de separabilidade indicaram que os fatores mão-de-obra, equipamentos e terra não podem ser agregados, pois a proporção destes seria alterada, dada à variação no preço de insumos. No entanto, o teste de separabilidade para as variáveis equipamentos e terra indicou que aqueles fatores são separáveis do fator insumos, mas as mudanças relativas da demanda desses insumos, decorrente dos choques aplicados no preço de insumos, indicam que houve alteração na proporção de utilização de terra e equipamentos. Apesar de não ser possível avaliar se esta variação foi estatisticamente diferente de zero, a contradição desses resultados requer que as conclusões relativas à separabilidade, baseadas no teste apresentado, sejam melhor estudadas.

\section{Conclusão}

Conclui-se que a desvalorização cambial provocou alterações na oferta do café, bem como no preço, na quantidade demandada dos fatores e nas parcelas de custo. Como os choques de uma desvalorização cambial foram aplicados nos preços de insumos e equipamentos, a mudança re- 
lativa observada na produção foi menor do que a do cenário em que se considerou o impacto apenas no preço do café. Ao contrário de Figueiredo et al. (1999) verificou-se que a mudança relativa na variável Equipamentos, quanto à quantidade demandada, foi relevante, o que reflete maior importância desta variável para o sistema de produção do Sul de Minas Gerais, onde a mecanização ocorre de forma mais intensiva.

Uma limitação deste trabalho foi a utilização de elasticidades-preço da oferta defasadas, que se fez necessário devido à falta de estimativas mais atuais. A sugestão para novos trabalhos é estimar as elasticidades de oferta para as principais culturas que possam refletir as condições atuais da produção.

Apesar de o efeito da desvalorização sobre o preço do produto e, conseqüentemente sobre a oferta ser menor quando se considera o impacto da desvalorização nos fatores, a desvalorização cambial estimulou a produção, ao tornar os produtos mais competitivos no mercado externo. Assim, na cultura do café, o câmbio desvalorizado estimulou a produção e aumentou a demanda de insumos, o que elevou a geração de empregos no campo. Para os choques considerados em ambos os cenários, o aumento percentual do fator mão-de-obra foi de 14,92\% .

Os resultados permitem inferir que o café foi beneficiado pela desvalorização cambial, embora tenha ocorrido aumento no preço dos insumos, o mesmo podendo acontecer para outras culturas de exportação.

\section{Referências bibliográficas}

ABIC - Associação Brasileira da Indústria do Café. Estatísticas. Disponível em: www.abic.com.br. Acessado em fev/2005.

CNA - Confederação Nacional da Agricultura. Indicadores Rurais. Disponível em: www.cna.org.br. Acessado em fev/2005.

COFFE BUSINESS. Anuário Estatístico do Café. Rio de Janeiro: 2001 COFFE BUSINESS. Anuário Estatístico do Café. Rio de Janeiro: 2004.

BERNDT, E.R.; CHRISTENSEN, L.R.; The translog function and the substitution of equipment, structures, and labor in US Manufacturing 1929-68. Journal of Econometrics, p. 81-114 , 1973. 


\section{EVIEWS - Quantitative Micro Software}

FIGUEIREDO, A.M.; FERREIRA, A.V.; TEIXEIRA, E. C. - Impactos de uma desvalorização cambial sobre as relações de produção na cultura do café. Revista de Economia e Sociologia Rural : 1999, 37(4), out/dez, p. $47-69$.

HERTEL, T. W. - Applications of duality and flexible functional forms: the case of multiproduct firm. Purdue: Purdue University, 1984 (Research Bulletin, 980).

ZYLBERSZTAJN, D. - Price distortions in brasilian agriculture: an application of duality theory on flexible functional forms. Raleigh: North Carolina State University, 1983, Ph. D. dissertation - North Carolina State University, 1983, 142 p.

Recebido em outubro de 2003 e revisto em junho de 2005 